\title{
A polygenic risk score predicts non-verbal intelligence in mentally healthy young adults
}

\author{
Kazantseva A. ${ }^{1,2 *}$, Enikeeva R. ${ }^{1}$, Davydova Yu. ${ }^{1}$, Mustafin R. ${ }^{3}$, Takhirova Z. ${ }^{1}$, \\ Mikhailova A. ${ }^{4}$, Lobaskova M. ${ }^{5}$, Malykh S. ${ }^{5,6}$, Khusnutdinova E. ${ }^{1,3,6}$ \\ ${ }^{1}$ Institute of Biochemistry and Genetics - Subdivision of the Ufa Federal Research Centre of the Russian \\ Academy of Sciences, Ufa, Russia \\ ${ }^{2}$ Ufa State Petroleum Technological University, Ufa, Russia \\ ${ }^{3}$ Bashkir State Medical University, Ufa, Russia \\ ${ }^{4}$ Bashkir State University, Ufa, Russia \\ ${ }^{5}$ Psychological Institute of Russian Academy of Education, Moscow, Russia \\ ${ }^{6}$ Lomonosov Moscow State University, Moscow, Russia \\ *Kazantsa@mail.ru
}

Key words: polygenic risk score, cognitive abilities, linear regression

Motivation and Aim: A polygenic risk score (PRS) approach directed toward identifying the best models explaining variance in different cognitive domains in European cohorts have been conducted. However, PRS studies of non-verbal intelligence (NVI) in Russian population remain scarce. The aim of the study was to estimate whether PRS based on SNPs and environmental parameters could predict individual differences in NVI in mentally healthy young adults from Russia.

Methods and Algorithms: The study included 1011 mentally healthy individuals (80\% women; $19.79 \pm 1.69$ years) of Caucasian origin from Russia (Russians, Tatars, Udmurts, and mixed ethnicity). NVI score was assessed via Raven's progressive matrices. Genotyping of examined 20 SNPs in the NGF rs63330, NRXN1 rs1045881, rs4971648, KIBRA rs17070145, NRG1 rs6994992, BDNF rs6265, GRIN2B rs3764030, APOE rs7412, rs429358, SNAP25 rs363050, IL1b rs16944, IL1A rs1800587, CRP rs3093077, TNF rs1041981, rs1800629, P2X7R rs2230912, C3AR1 rs7842, DNMT1 rs10418707, DNMT3a rs1550117, DNMT3b rs2424932 genes was performed using PCR-based KASP genotyping. The sample was divided into training $(N=711)$ and testing sets $(N=300)$. Statistical analysis included multiple linear regressions with genotypes and various social parameters (specificity of child-parent relationships) as predictors for the training sample. PRS assessment was conducted in the testing sample with linear regression (PLINK v.1.09, R).

Results: A series of linear regression analysis identified the best PRS model ( $\beta=0.79$; $\left.P=0.031, \mathrm{r}^{2}{ }_{\text {adj }}=2.02 \%\right)$, which was based on the inclusion of minor alleles of all examined SNPs, sex $(\beta=-0.86 ; P=0.082)$, ethnicity $(\beta=1.32 ; P=0.072)$ and bilingual rearing $(\beta=-0.65 ; P=0.287)$ and explained about $2 \%$ of variance in non-verbal intelligence. According to identified model, men and individuals without bilingual style of rearing (in non-Russian ethnic groups) demonstrated higher NVI.

Conclusion: Our findings established the best PRS model based on genetic and environmental predictors, which successfully explained $\sim 2 \%$ of individual differences in non-verbal intelligence in young adults from Russia.

Acknowledgements: The study is supported by the Russian Science Foundation (17-7830028). 